International Journal of Modern Physics E (C) World Scientific Publishing Company

\title{
CHIRAL UNITARY DYNAMICS OF HADRONS AND HADRONS IN A NUCLEAR MEDIUM
}

\author{
* \\ E. OSET, L. S. GENG, D. GAMERMANN, M.J. VICENTE VACAS, D. STROTTMAN, K. P. \\ KHEMCHANDANI, A. MARTINEZ TORRES ${ }^{\dagger}$ \\ Departamento de Física Teórica and IFIC, Centro Mixto Universidad de Valencia-CSIC, \\ Institutos de Investigación de Paterna \\ Aptd. 22085, 46071 Valencia, Spain. ${ }^{\ddagger}$ \\ J. A. OLLER AND L. ROCA \\ Departamento de Física. Universidad de Murcia. \\ Murcia. E-30071 Murcia. Spain. \\ MAURO NAPSUCIALE \\ Instituto de Fíisica, Universidad de Guanajuato \\ Lomas del Bosque 103, Fraccionamiento Lomas del Campestre \\ 37150, León Guanajuato, México. \\ Received (received date) \\ Revised (revised date)
}

\begin{abstract}
In this talk I summarize recent findings around the description of axial vector mesons as dynamically generated states from the interaction of pseudoscalar mesons and vector mesons, dedicating some attention to the two $K_{1}(1270)$ states. Then I review the generation of open and hidden charm scalar and axial states, and how some recent experiment supports the existence of the new hidden charm scalar state predicted. I present recent results showing that the low lying $1 / 2^{+}$baryon resonances for $\mathrm{S}=-1$ can be obtained as bound states or resonances of two mesons and one baryon in coupled channels. Then show the differences with the $\mathrm{S}=0$ case, where the $N^{*}(1710)$ appears also dynamically generated from the two pion one nucleon system, but the $N^{*}(1440)$ does not appear, indicating a more complex structure of the Roper resonance. Finally I shall show how the state $\mathrm{X}(2175)$, recently discovered at BABAR and BES, appears naturally as a resonance of the $\phi K \bar{K}$ system.
\end{abstract}

\section{Introduction}

The combination of nonperturbative unitary techniques in coupled channels with the QCD information contained in the chiral Lagrangians has allowed one to ex-

\footnotetext{
*chiral unitary dynamics of hadrons and hadrons in a nuclear medium †oset@ific.uv.es

$\ddagger$
} 
tend the application domain of traditional Chiral Perturbation theory to a much larger range of energies where many low lying meson and baryon resonances appear. For instance, for the interactions between the members of the lightest octet of pseudoscalars, one starts with the chiral Lagrangian of ref. ${ }^{112}$ and selects the set of channels that couple to certain quantum numbers. Then, independently of using either the Bethe-Salpeter equation in coupled channels 3 , the N/D method 4 or the Inverse Amplitude Method 5 , the well known scalar resonances $\sigma(600)$, $f_{0}(980), a_{0}(980)$ and $\kappa(800)$ appear as poles in the obtained $\mathrm{L}=0$ meson-meson partial waves. These resonances are not introduced by hand, they appear naturally as a consequence of the meson interaction and they qualify as ordinary bound states or resonances in coupled channels. These are states that we call dynamically generated, by contrast to other states which would rather qualify as $q \bar{q}$ states, such as the $\rho$. Similarly, in the baryon sector, the interaction of the pseudoscalar mesons with baryons of the octet of the proton generates dynamically $1 / 2^{-}$resonances $78|9| 10|11| 12$ and the interaction of the pseudoscalar mesons with baryons of the decuplet of the $\Delta$ generates $3 / 2^{-}$resonances $13[14$. These last two cases can be unified using $\mathrm{SU}(6)$ symmetry as done in 15 . This field has proved quite productive and has been further extended by combining pseudoscalar mesons with vector mesons, which lead to the dynamical generation of axial vector mesons like the $a_{1}(1260), b_{1}(1235)$, etc 1617. Also in the charm sector one has obtained in this way scalar mesons with charm, like the $D_{s 0}(2317)$ [18/19]20|21, axial vector mesons with charm like the $D_{s 1}(2460) 16|22| 23$, or hidden charm scalars like a predicted $X(3700)$ state and two hidden charm axial states, with opposite C-parity, one of which corresponds to the $X(3872)$ state. In what follows we briefly discuss these latter cases.

Paralelly with these developments investigation has started regarding systems with three hadrons not studied so far, like two mesons and a baryon, or three mesons. A new formalism is developed, based on the Faddeev equations and the on shell scattering amplitudes of the different components. This can be done because one can prove that there are cancellations between the off shell part of these amplitudes and the three body contact forces that originate from the same chiral lagrangians. This novel finding should stimulate thoughts around conventional Faddeev equations which rely upon the full off shell extrapolation of the amplitudes, and eventually require some three body forces that must be put by hand to reproduce the data. I shall report on several states which are obtained within this new formalism and which can be associated to well known resonances.

\section{Axial vector mesons dynamically generated}

As shown in detail in 16|17, starting from a standard chiral Lagrangian for the interaction of pseudoscalar mesons of the octet of the $\pi$ and vector mesons of the octet of the $\rho$, and unitarizing in coupled channels solving the coupled BetheSalpeter equations, one obtains the scattering matrix for pseudoscalar mesons with 
vectors for different quantum numbers, which contains poles that can be associated to known resonances like the $a_{1}(1260), b_{1}(1235)$, etc. The $\mathrm{SU}(3)$ decomposition of $8 \times 8$

$$
8 \times 8=1+8_{s}+8_{a}+10+\overline{10}+27
$$

leads here to two octets, unlike in the case of the interaction of pseudoscalars among themselves where there is only room for the $8_{s}$ representation. This is why here one finds different G-parity states like the $a_{1}$ and $b_{1}$, the $f_{1}(1285), h_{1}(1380)$, plus an extra $h_{1}(1170)$ that one can identify with the singlet state. One should then find two $K_{1}$ states, which do not have defined G-parity. One might think that these states are the $K_{1}(1270)$ and the $K_{1}(1400)$ states. However the theory fails to predict a state with such a large mass as the $K_{1}(1400)$ and with its decay properties. Instead, in 17 two states were found with masses close by, given, after some fine tuning, by 1197 $\mathrm{MeV}$ and $1284 \mathrm{MeV}$, and widths of about $240 \mathrm{MeV}$ and $140 \mathrm{MeV}$, respectively 24. The interesting thing about these states is that the first one couples most strongly to $K^{*} \pi$, while the second state couples most strongly to $K \rho$. One could hope that these two states could be observed experimentally. Indeed, this is the case as was shown in the recent work ${ }^{24}$ by looking at two reactions which have either $K^{*} \pi$ or $K \rho$ in the final state and which clearly show the peak at different positions, as one can observe in fig. 1.

It is interesting to recall that in the experimental analysis done in 25 only one $K_{1}(1270)$ resonance was included (together with the $K_{1}(1400)$ which shows up at higher energies), but the background was very large and the peaks appeared from interference of large background terms rather than from the effect of the resonance. Instead in 24, with the introduction of the two resonances obtained in our approach and the background generated by the same chiral unitary approach, together with a contribution from the $K_{1}(1400)$ considered phenomenologically, the description of all data in fig. 1 follows in a natural way.

\section{Dynamically generated scalar mesons with open and hidden charm}

A generalization to $\mathrm{SU}(4)$ of the $\mathrm{SU}(3)$ chiral Lagrangian for meson-meson interaction is done in 21 to study meson-meson interaction including charm. The breaking of $\mathrm{SU}(4)$ is done as in 26/27, where the crossed exchange of vector mesons is employed as it accounts phenomenologically for the Weinberg-Tomozawa term in the chiral Lagrangians. With this in mind, when the exchange is due to a heavy vector meson the corresponding term is corrected by the ratio of square masses of the light vector meson to the heavy one (vectors with a charmed quark). We also use a different pattern of SU(4) symmetry breaking by following the lines of a chiral motivated model with general $\mathrm{SU}(\mathrm{N})$ breaking 28 . The picture generalizes the model used in $18|19| 20$, where only the light vector mesons are exchanged. The same states generated in $\frac{1320}{20}$ are also generated in 21 with some changes, but in addition one 

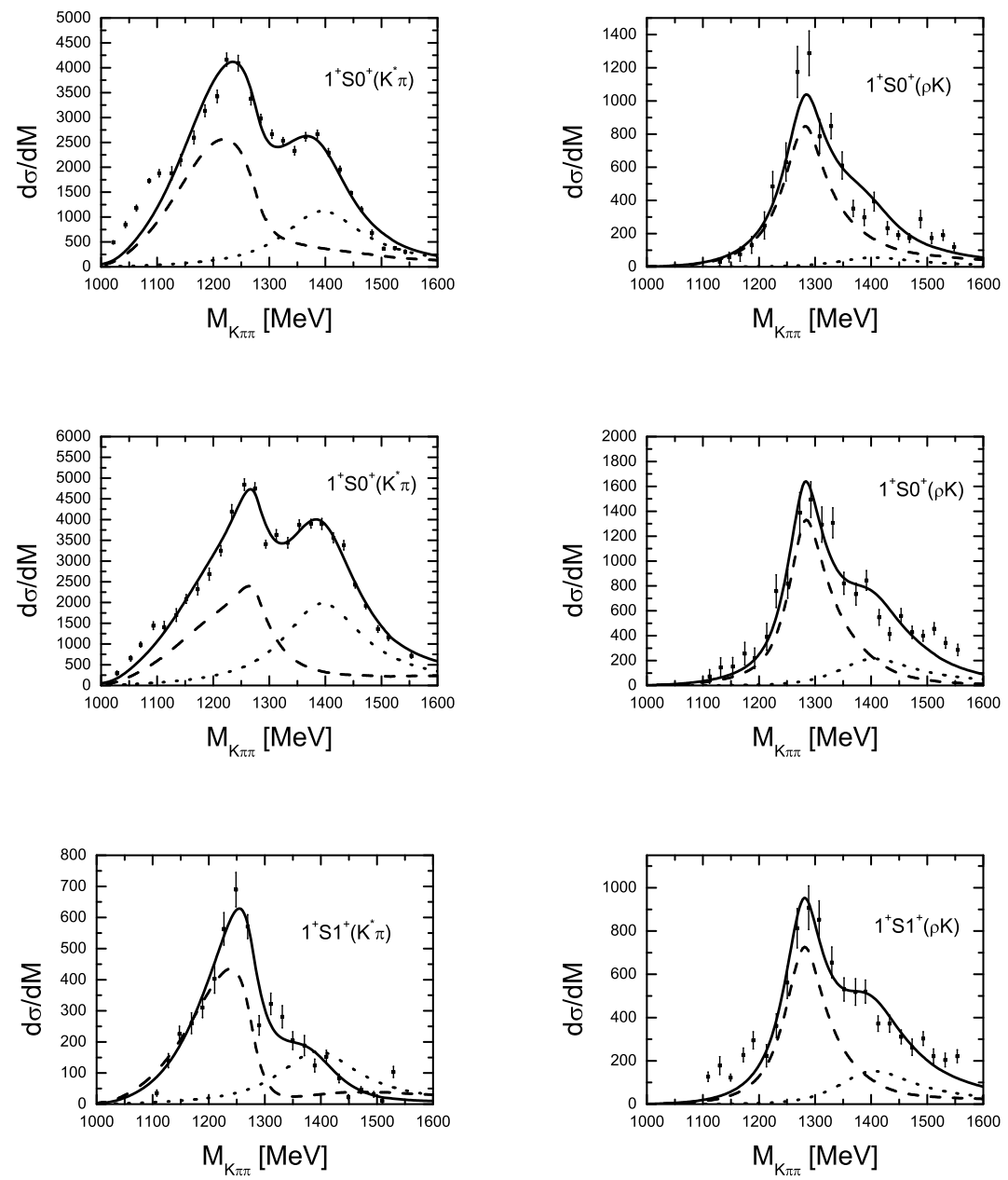

Fig. 1. Results for the $\pi \pi K$ invariant mass distribution in the $K^{-} p \rightarrow K^{-} \pi^{+} \pi^{-} p$ reaction. Data in the upper panels are for $0 \leq\left|t^{\prime}\right| \leq 0.05 \mathrm{GeV}^{2}$ and those in the middle and bottom panels for $0.05 \leq\left|t^{\prime}\right| \leq 0.7 \mathrm{GeV}^{2}$, where $t^{\prime}$ is the four momentum transfer squared to the recoiling proton. The data are further grouped by $J^{P} L M^{\eta}$ followed by the isobar and odd particle. $\mathrm{J}$ is the total angular momentum, $\mathrm{P}$ the parity, L the orbital angular momentum of the odd particle. $M^{\eta}$ denotes the magnetic substate of the $K \pi \pi$ system and the naturality of the exchange.

obtains states with hidden charm. The changes refer to the states of the sextet, which in 21 appear rather broad, while these are narrow in other works. In table 1 we show the states with charm or hidden charm obtained in the present approach.

As we can see, the $D_{s 0}(2317)$ and $D_{0}(2400)$ appear in the approach, the last one at a lower energy than in experiment, but consistent with the data considering 
Table 1: Pole positions for the model. The column Irrep shows the results in the $S U(3)$ limit.

\begin{tabular}{c|c||c|c|c|c|c}
\hline $\mathrm{C}$ & $\begin{array}{c}\text { Irrep } \\
\text { Mass }(\mathrm{MeV})\end{array}$ & $\mathrm{S}$ & $\mathrm{I}\left(J^{P}\right)$ & $\mathrm{RE}(\sqrt{s})(\mathrm{MeV})$ & $\mathrm{IM}(\sqrt{s})(\mathrm{MeV})$ & Resonance ID \\
\hline \hline \multirow{3}{*}{1} & \multirow{3}{*}{$\overline{3}$} & 1 & $0\left(0^{+}\right)$ & 2317.25 & 0 & $D_{s 0}^{*}(2317)$ \\
\cline { 2 - 7 } & 2327.96 & 0 & $\frac{1}{2}\left(0^{+}\right)$ & 2129.26 & -157.00 & $D_{0}^{*}(2400)$ \\
\cline { 2 - 7 } & 6 & 1 & $1\left(0^{+}\right)$ & 2704.31 & -459.50 & $(?)$ \\
\cline { 2 - 7 } & 2394.87 & 0 & $\frac{1}{2}\left(0^{+}\right)$ & 2694.69 & -441.89 & $(?)$ \\
\cline { 2 - 7 } & $-\mathrm{i} 219.33$ & -1 & $0\left(0^{+}\right)$ & 2709.39 & -445.73 & $(?)$ \\
\hline 0 & 1 & 0 & $0\left(0^{+}\right)$ & 3718.93 & -0.06 & \\
\hline
\end{tabular}

the large width of the state and the theoretical and experimental uncertainties on the mass. The other three charm states in the table come from a sextet and they are very broad in our approach $(\Gamma \sim I M(\sqrt{s}))$.

The very interesting and novel aspect with respect to other theoretical works is the heavy state with zero charm. It is a hidden charm state mostly built from $D \bar{D}$ and $D_{s} \bar{D}_{s}$. The fact that this state has such a narrow width in spite of having all the meson-meson states of the light sector open for decay, is an interesting consequence of the work, which largely decouples the light sector from the heavy one respecting basic OZI rules. There is no experimental information on this state presently, but an enhancement of the cross section of the $e^{+} e^{-} \rightarrow J / \psi D \bar{D}$ close to the $D \bar{D}$ threshold seen in 29 could be interpreted 30 as a consequence of the effect of the $\mathrm{X}(3700)$, which is a bound state below, but close to, the $D \bar{D}$ threshold. I show this in detail in the next section.

\section{Experimental hints of the $\mathrm{X}(3700)$ hidden charm state}

The reaction $e^{+} e^{-} \rightarrow J / \psi D \bar{D}$ can be described by the diagram in fig. 2 if one assumes that the $D \bar{D}$ pair comes from a resonance.

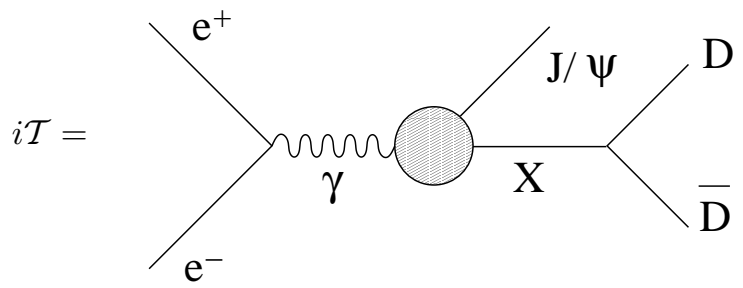

Fig. 2. Feynman diagram for the process $e^{+} e^{-} \rightarrow J / \psi D \bar{D}$ 
Close to threshold the only part of this amplitude which is strongly energy dependent is the $X$ propagator and all other parts can be factorized, so that we can write

$$
\mathcal{T}=C \frac{1}{M_{i n v}^{2}(D \bar{D})-M_{X}^{2}+i \Gamma_{X} M_{X}}
$$

if we describe the $X$ resonance as a Breit-Wigner type.

The cross section would then be given by an integral over the phase space of the three particles in the final state 30 .

Assuming that $\mathcal{T}$ depends only on the $D \bar{D}$ invariant mass, one can evaluate the differential cross section:

$$
\frac{d \sigma}{d M_{\text {inv }}(D \bar{D})}=\frac{1}{(2 \pi)^{3}} \frac{m_{e}^{2}}{s \sqrt{s}}|\vec{k}\|\vec{p}\| \mathcal{T}|^{2}
$$

where $s$ is the center of mass energy of the electron positron pair squared and $|\vec{k}|$ and $|\vec{p}|$ are given by:

$$
\begin{aligned}
& |\vec{p}|=\frac{\lambda^{1 / 2}\left(s, M_{J / \psi}^{2}, M_{i n v}^{2}(D \bar{D})\right)}{2 \sqrt{s}} \\
& |\vec{k}|=\frac{\lambda^{1 / 2}\left(M_{i n v}^{2}(D \bar{D}), M_{D}^{2}, M_{\bar{D}}^{2}\right)}{2 M_{i n v}(D \bar{D})}
\end{aligned}
$$

Where $\lambda^{1 / 2}\left(s, m^{2}, M^{2}\right)$ is the usual Källen function.

In the following we explain how we compare our results to Belle's data.

Belle has measured the differential cross section for $J / \psi D \bar{D}, J / \psi D \bar{D}^{*}$ and $J / \psi D^{*} \bar{D}^{*}$ production from electron positron collision at center of mass energy $\sqrt{s}=10.6 \mathrm{GeV} 29$. We are going to study the first case, where the scalar hidden charm state generated in the model of ${ }^{21}$ plays a special role. The Belle's measurement produces invariant mass distributions for the $D \bar{D}$ that range from threshold up to $5.0 \mathrm{GeV}$. Our model is in principle reliable for energies within few hundreds of $\mathrm{MeV}$ from the thresholds, so we compare numerically our results with the data up to $4.2 \mathrm{GeV}$.

The experiment measures counts per bin. In the case of a $D \bar{D}$ pair, the bins have $50 \mathrm{MeV}$ width, while for the $D \bar{D}^{*}$ pair they have $25 \mathrm{MeV}$. To compare the shape of our theoretical calculation with the experimental data we integrate our theoretical curve in bins of the same size as the experiment and normalize our results so that the total integral of our curve matches the total number of events measured in the invariant mass range up to $4.2 \mathrm{GeV}$. The comparison is made by the standard $\chi^{2}$ test.

As described in 21/23, in the heavy sector the model to evaluate the scattering Tmatrix has one free parameter, $\alpha_{H}$ which is the subtraction constant in the loop for channels with at least one heavy particle. In these previous papers this parameter 
Table 1. Results of $M_{X}$ and $\chi^{2}$ for different values of $\alpha_{H}$.

\begin{tabular}{c|cc}
\hline$\alpha_{H}$ & $M_{X}(\mathrm{MeV})$ & $\frac{\chi^{2}}{d . o . f}$ \\
\hline \hline-1.4 & $3701.93-\mathrm{i} 0.08$ & 0.96 \\
-1.3 & $3718.93-\mathrm{i} 0.06$ & 0.85 \\
-1.2 & $3728.12-\mathrm{i} 0.03$ & 0.92 \\
-1.1 & Cusp & 1.11 \\
\hline
\end{tabular}

has been fitted so that the pole in the $\mathrm{C}=1, \mathrm{~S}=1, \mathrm{I}=0$ sector matches the observed state with these quantum numbers $\left(D_{s 0}(2317)\right.$ and $D_{s 1}(2460)$ for scalar and axial states, respectively). The channels in this sector have always one heavy and one light meson, and in principle one could fit a different $\alpha$ for channels with two heavy particles. Here we are going to present results for different values of $\alpha$ in channels with hidden charm (doubly heavy channels). Since we are working with the $\mathrm{C}=0$ sector, we have also channels involving only light mesons. These have negligible influence in the pole position of the hidden charm poles as shown in 21 , so we leave $\alpha_{L}$ constant. The values chosen for $\alpha_{H}$ correspond to the natural size $\underline{9}$. In terms of an equivalent cut off to regularize the loop functions, the value $\alpha_{H}=-1.3$ corresponds to $q_{\max } \sim 850 \mathrm{MeV}$, for two $D$ mesons in a loop. As mentioned above, the values of $\alpha_{H}$ taken in the calculation correspond to those taken in 23|21. We have taken a range of $\alpha_{H}$ roughly around the values $\alpha_{H}=-1.3$ chosen for the scalar mesons 21 .

In table 1 we show results, for different values of $\alpha_{H}$, of the pole position of the hidden charm resonance in the scalar sector, and the value of $\chi^{2}$ calculated with the data from Belle, with combinatorial background already subtracted, for all points below $4.2 \mathrm{GeV}$ in the $J / \psi D \bar{D}$ production. Fig. 3 shows plots of our theoretical histograms compared with experimental data 29. Note that although we are plotting all points until $5.0 \mathrm{GeV}$, only the ones below 4.2 have been used in the calculation of $\chi^{2}$ and in the normalization of the theoretical curves.

The $\chi^{2}$ values obtained in table 1 are around 1 or below, indicating a good fit to the data in all curves. This is in part due to the large experimental errors, but the clear message is that the presence of a pole below the $D \bar{D}$ threshold is enough to reproduce the observed enhancement of the cross section for this reaction in the $D \bar{D}$ invariant mass above threshold. The results of table 1 and inspection of fig. 3 show some preference for values of $\alpha_{H}=-1.3,-1.2$, which would correspond to the hidden charm scalar with mass slightly above $3700 \mathrm{MeV}$.

The peak seen in the experiment has been fitted with a Breit-Wigner like resonance in 29 , suggesting a new resonance. In order to make the results obtained here more meaningful, we also perform such a fit and compare the results. We take the same Breit-Wigner parameters suggested in the experimental paper, $M_{X}=3878$ $\mathrm{MeV}$ and $\Gamma_{X}=347 \mathrm{MeV}$. We show the results obtained by fitting a Breit-Wigner 

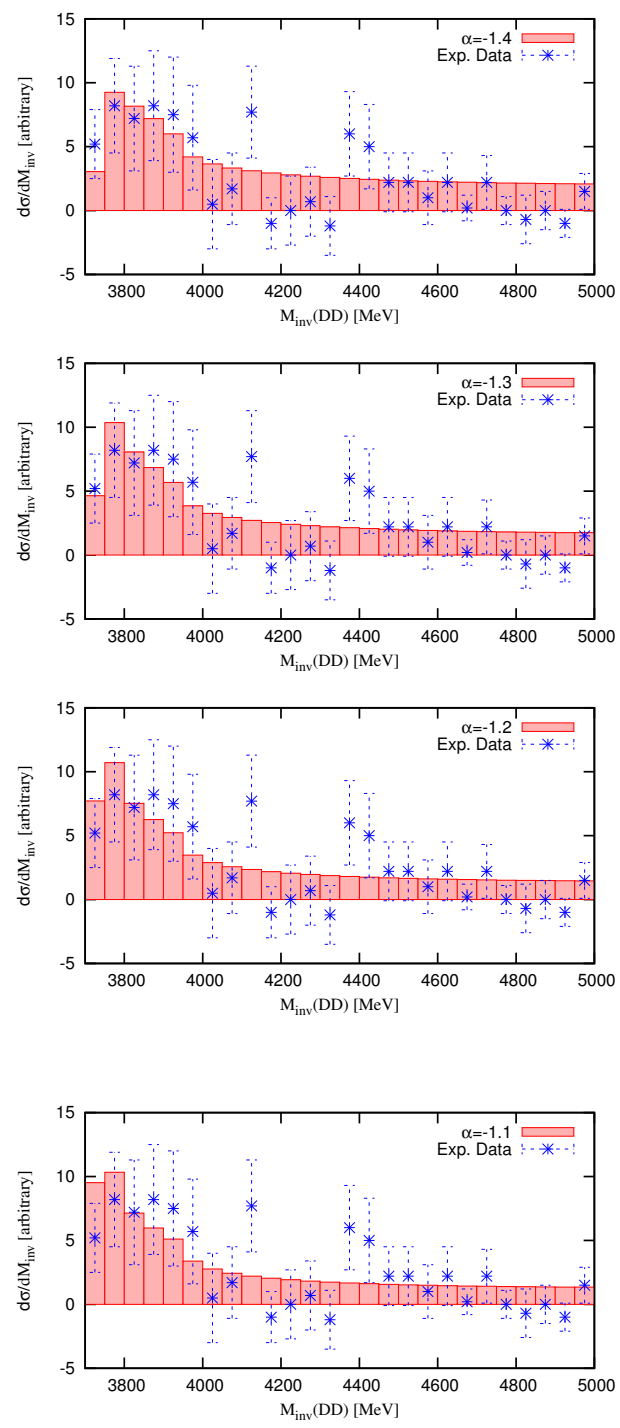

Fig. 3. Theoretical histograms compared with data for $D \bar{D}$ invariant mass distribution.

form from eq. (2) in $\mathcal{T}$ of eq. (3) in fig. 4. Additionally we calculate $\chi^{2}$ and find $\chi^{2} /$ d.o. $f=2.10$ for the $D \bar{D}$ distribution. The value of $\chi^{2}$ for the $D \bar{D}$ distribution can be improved if we take different parameters for the Breit-Wigner resonance. Taking for the fit $M_{X}=3750 \mathrm{MeV}$ and $\Gamma_{X}=250 \mathrm{MeV}$ we obtain a value of $\chi^{2} /$ d.o. $f=1.12$, still slightly bigger than those obtained in our previous analysis assuming the mechanism of fig. 2 driven by the $X(3700)$ scalar state. 
As a consequence of the discussion, our conclusions would be that for the case of the broad peak seen in $D \bar{D}$, the weak case in favor of a new state around 3880 $\mathrm{MeV}$ discussed in 29 is further weakened by the analysis done here, showing that the results are compatible with the presence of a scalar hidden charm state with mass around $3700 \mathrm{MeV}$.

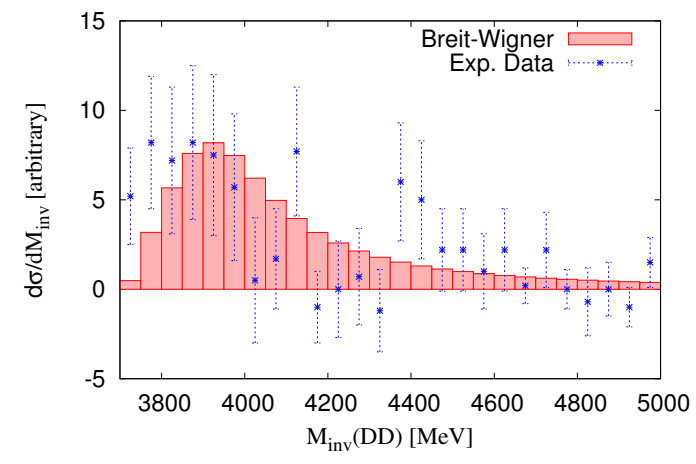

Fig. 4. Histograms calculated with Breit-Wigner resonance with mass $M_{X}=3880 \mathrm{MeV}$ compared to data.

\section{Dynamically generated axial vector mesons with open and hidden charm}

With the interaction of pseudoscalar mesons with vector mesons in 23 one obtains the results shown in Table 2. In addition to the well known $D_{s 1}(2460), D_{1}(2430)$, $D_{s 1}(2536)$ and $D_{1}(2420)$ (and all those in the light sector already found in 16/17) one obtains new states, which could be observed, although some of them are either too broad or correspond to cusps.

Very interesting and novel in the present approach is the generation of the $\mathrm{X}(3872)$ with positive C-parity and another state nearly degenerate with negative C-parity. It would be interesting to see if a state with negative C-parity is observed, but the large branching fraction

$$
\frac{B\left(X \rightarrow \pi^{+} \pi^{-} \pi^{0} J / \psi\right)}{B\left(X \rightarrow \pi^{+} \pi^{-} J / \psi\right)}=1.0 \pm 0.4 \pm 0.3
$$

indicates either a very large G-parity (isospin) violation (quite unlikely), or the existence of another state with different C-parity (G-parity also in this case). 
Table 2: Pole positions for the model. The column Irrep shows the results in the $S U(3)$ limit. The results in brackets for the $\operatorname{Im} \sqrt{s}$ are obtained taking into account the finite width of the $\rho$ and $K^{*}$ mesons.

\begin{tabular}{|c|c|c|c|c|c|c|}
\hline $\mathrm{C}$ & $\begin{array}{c}\text { Irrep } \\
\text { Mass }(\mathrm{MeV})\end{array}$ & $\mathrm{S}$ & $\mathrm{I}^{G}\left(J^{P C}\right)$ & $\begin{array}{c}\mathrm{RE}(\sqrt{s}) \\
(\mathrm{MeV})\end{array}$ & $\begin{array}{c}\mathrm{IM}(\sqrt{s}) \\
(\mathrm{MeV}) \\
\end{array}$ & Resonance ID \\
\hline \multirow[t]{10}{*}{1} & \multirow{2}{*}{$\begin{array}{c}\overline{3} \\
2432.63\end{array}$} & 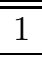 & $0\left(1^{+}\right)$ & 2455.91 & $\overline{0}$ & $\overline{D_{s 1}(2460)}$ \\
\hline & & 0 & $\frac{1}{2}\left(1^{+}\right)$ & 2311.24 & -115.68 & $D_{1}(2430)$ \\
\hline & \multirow{3}{*}{$\begin{array}{c}6 \\
2532.57 \\
-\mathrm{i} 199.36\end{array}$} & 1 & $1\left(1^{+}\right)$ & 2529.30 & -238.56 & $(?)$ \\
\hline & & 0 & $\frac{1}{2}\left(1^{+}\right)$ & Cusp (2607) & Broad & $(?)$ \\
\hline & & -1 & $0\left(1^{+}\right)$ & Cusp (2503) & Broad & $(?)$ \\
\hline & \multirow{2}{*}{$\begin{array}{c}\overline{3} \\
2535.07 \\
-\mathrm{i} 0.08\end{array}$} & 1 & $0\left(1^{+}\right)$ & 2573.62 & $-0.07[-0.07]$ & $D_{s 1}(2536)$ \\
\hline & & 0 & $\frac{1}{2}\left(1^{+}\right)$ & 2526.47 & $-0.08[-13]$ & $D_{1}(2420)$ \\
\hline & \multirow{3}{*}{$\begin{array}{c}6 \\
\text { Cusp (2700) } \\
\text { Narrow }\end{array}$} & 1 & $1\left(1^{+}\right)$ & 2756.52 & -32.95 [cusp] & $(?)$ \\
\hline & & 0 & $\frac{1}{2}\left(1^{+}\right)$ & 2750.22 & $-99.91[-101]$ & $(?)$ \\
\hline & & $\overline{-1}$ & $0\left(1^{+}\right)$ & 2756.08 & $-2.15[-92]$ & $(?)$ \\
\hline \multirow[t]{2}{*}{0} & $\begin{array}{c}1 \\
3867.59\end{array}$ & 0 & $0^{+}\left(1^{++}\right)$ & 3837.57 & $\begin{array}{l}-0.00 \\
\end{array}$ & $X(3872)$ \\
\hline & $\begin{array}{c}1 \\
3864.62\end{array}$ & 0 & $0^{-}\left(1^{+-}\right)$ & 3840.69 & -1.60 & $(?)$ \\
\hline
\end{tabular}

\section{Dynamically generated $1 / 2^{+}$baryon states from the interaction of two mesons and one baryon}

We discussed before how the low lying $1 / 2^{-}$baryon resonances appear dynamically generated in the chiral unitary approach. The low lying $1 / 2^{+}$resonances are not less problematic and quark models have difficulties in reproducing them 31 . Experimentally some of them are poorly understood and few of them possess four-star status. Among the rest some resonances are listed with unknown spin parity and two are controversial in nature. The situation is slightly better with the $\Lambda$ resonances in the same energy region, except for the $\Lambda(1600)$ and $\Lambda(1810)$, where the peak positions and widths, obtained by different partial wave analysis groups, vary a lot. Many of these $S=-1$ states seem to have significant branching ratios for three-body, i.e., two meson-one baryon, decay channels. However, no theoretical attempt has been made to study the three body structure of these resonances, until recently when a coupled channel calculation for two meson one baryon system was carried out using chiral dynamics 3233 . 


\section{Formalism for the three body systems}

We take advantage of the fact that there are strong correlations in the meson baryon sector in $\mathrm{L}=0$, and with $\mathrm{S}=-1$ one obtains many $1 / 2^{-}$resonances. The $\Lambda(1405) S_{01}$ $\left(J^{P}=1 / 2^{-}\right)$couples strongly to the $\pi-\Sigma$ and its coupled channels. Considering this we build the three body coupled channels by adding a pion to combinations of a pseudoscalar meson of the $0^{-} \mathrm{SU}(3)$ octet and a baryon of the $1 / 2^{+}$octet which couple to $S=-1$. For the total charge zero of the three body system we get twenty-two coupled channels. Details can be seen in $32 \mid 33$.

To solve the Faddeev equations we write the two body $t$-matrices using unitary chiral dynamics. These $t$-matrices can be split into an on-shell part, depending only on the respective center of mass energy, and an off-shell part, which is inversely proportional to the propagator of the off-shell particle. This off-shell part cancels a propagator in the three body scattering diagrams, leading to a diagram with a topological structure equivalent to that of a three body force 32|33. To this, one must add the three body forces originating directly from the chiral Lagrangians. Interestingly, in our case, we find that the three body forces from the two sources cancel in the $\mathrm{SU}(3)$ limit. In a realistic case, we find them to sum-up to merely $5 \%$ of the total on-shell contribution of the $t$-matrices to the Faddeev equations. The formalism is thus developed further in terms of the on-shell parts of the two body $t$-matrices.

We begin with Faddeev equations

$$
T^{i}=t^{i} \delta^{3}\left(\vec{k}_{i}^{\prime}-\vec{k}_{i}\right)+t^{i} g^{i j} T^{j}+t^{i} g^{i k} T^{k},
$$

which, if iterated while neglecting the terms with $\delta^{3}\left(\vec{k}_{i}^{\prime}-\vec{k}_{i}\right)$, corresponding to the disconnected diagrams, will give

$T^{i}=t^{i} g^{i j} t^{j}+t^{i} g^{i k} t^{k}+t^{i} g^{i j} t^{j} g^{j k} t^{k}+t^{i} g^{i j} t^{j} g^{j i} t^{i}+t^{i} g^{i k} t^{k} g^{k j} t^{j}+t^{i} g^{i k} t^{k} g^{k i} t^{i}+\ldots$

In order to convert the Faddeev equations into a set of algebraic equations one writes the terms with three successive interactions explicitly, which already involve a loop evaluation. One finds technically how to go from the diagrams with two interactions to those with three interactions and the algorithm found is then used for the next iterations, leading thus to a set of algebraic equations, which are solved within twenty two coupled channels.

The resulting Faddeev equations have been solved with the input two body $t$ matrices obtained by solving the Bethe-Salpeter equation as in $3|8| 34$. We find four $\Sigma$ and two $\Lambda$ states as dynamically generated resonances from a two meson-one baryon system, which we associate to known resonances of the PDG 35 , implying a strong coupling of the $S=-1$ resonances, in this region, to the three body decay channels. In Fig. 5 we plot the modulus square of the T-matrix as a function of two variables, the total energy and the invariant mass of particles two and three. We show results for one of the resonances, corresponding to the $\Sigma(1660) 35$ found in our study in the squared amplitude for the $\pi^{0} \pi^{0} \Sigma^{0}$ channel, and in fig. 6 we show the results for the $\Lambda(1600)$. In addition to this, we find evidence for (1) another 
$1 / 2^{+}$resonance, i.e., the $\Sigma(1770)$, (2) for the controversial $\Sigma(1620)$ and (3) for the $\Sigma(1560)$, which is listed with unknown spin-parity 35 . In the isospin 0 sector we find evidence for the $\Lambda$ (1600) and $\Lambda$ (1810).

In a recent paper ${ }^{36}$ the work has been extended to the sector with $S=0$. There one finds neatly the $N^{*}(1710)$ as a resonant state of $\pi \pi N$, but the Roper $N^{*}(1440)$ does not show up, indicating a far more complicated structure, as one may

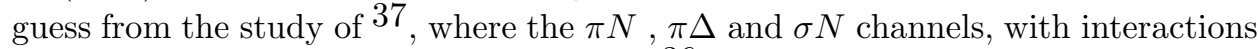
additional to those used in the approach of $\frac{36}{a}$ are considered. In this paper one can find in detail the cancellations between the off shell parts of the amplitudes and the three body forces coming from the chiral Lagrangians that we have mentioned above. The signal for the $N^{*}(1710)$ is seen in fig. 7 .

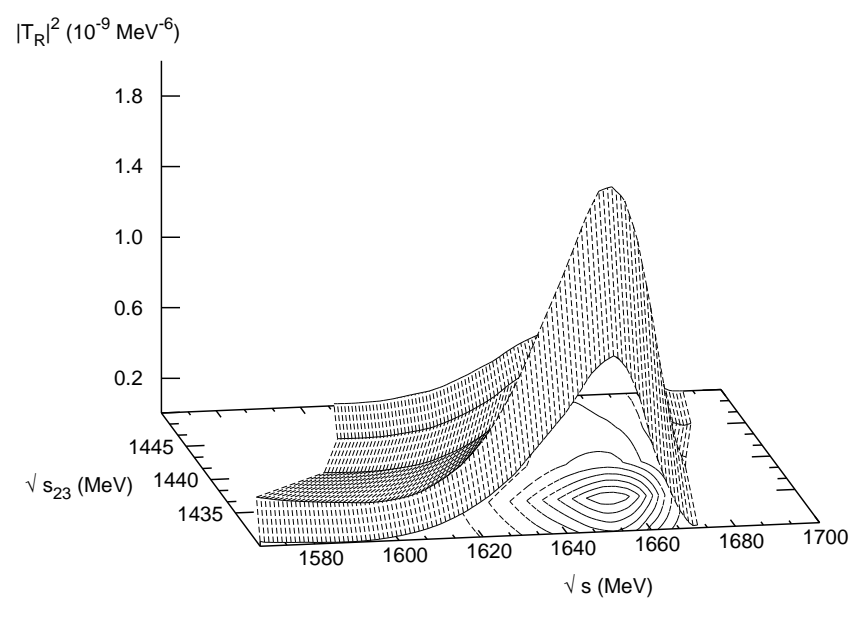

Fig. 5. The $\Sigma(1660)$ resonance in the $\pi^{0} \pi^{0} \Sigma^{0}$ channel.

Finally we would like to report on the recent work on the generation of the

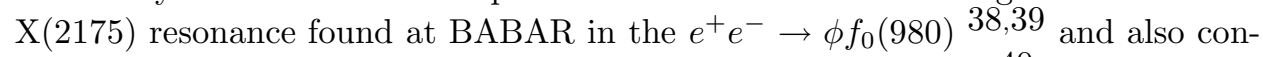
firmed at BES in the study of the $J / \psi \rightarrow \eta \phi f_{0}(980)$ decay mode 40 . The work has been done in 41 with the same three body scheme using the particles $\phi K \bar{K}$. A neat resonance is found around $2150 \mathrm{MeV}$ with a width compatible with experiment, see fig. 8. As mentioned above, we plot the modulus square of the $T$ matrix as a function of two variables, the total energy and the invariant mass of the $K \bar{K}$ subsystem. We not only find the mass on the right place, but we also find the peak of the $K \bar{K}$ invariant mass at the $f_{0}(980)$ position, indicating that the system of three particles is strongly correlated in a $\phi$ and the $f_{0}(980)$ as found in the experiment. 


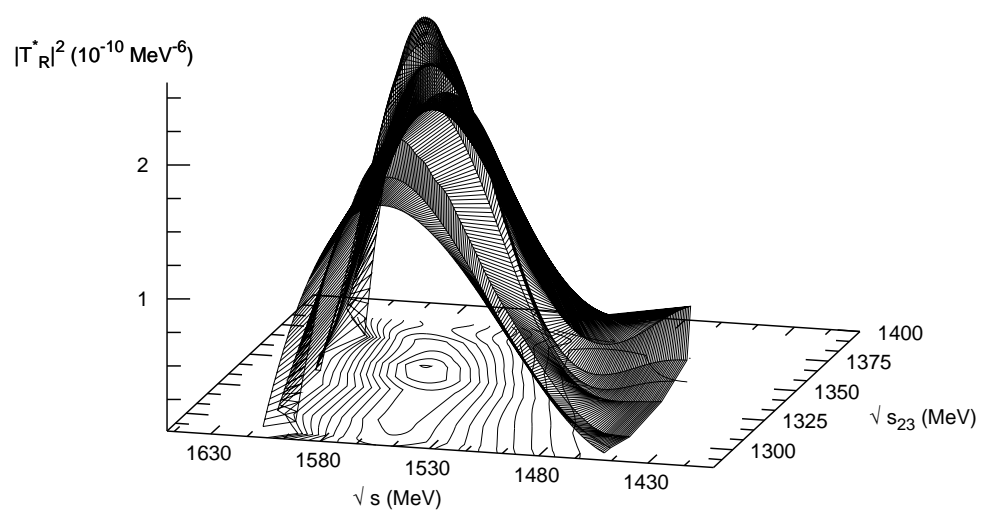

Fig. 6. A $\Lambda$ resonance in the $\pi \bar{K} N$ amplitude at $1568 \mathrm{MeV}$ in $I=0, I_{\pi \bar{K}}=1 / 2$.

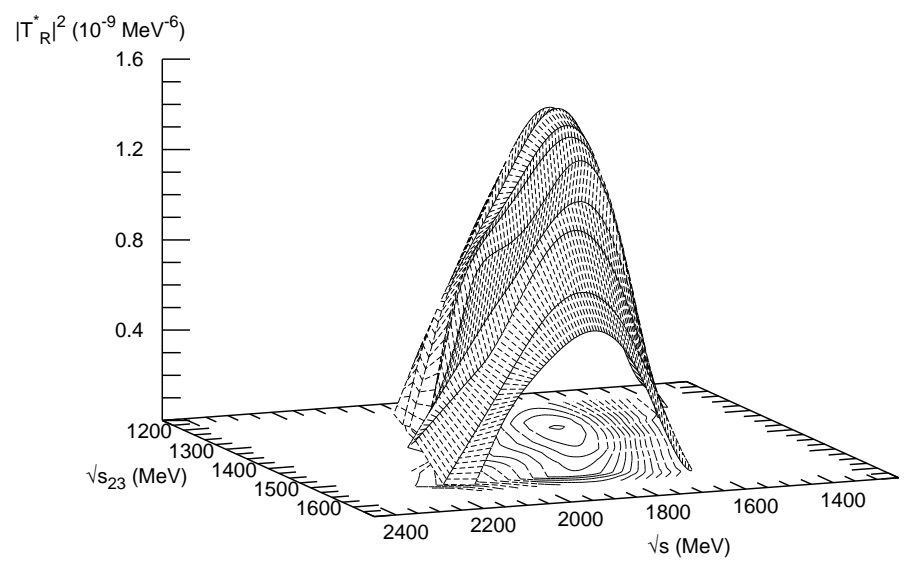

Fig. 7. The squared amplitude for the $\pi \pi N$ system in isospin $1 / 2$ configuration as a function of $\sqrt{s}$ and $\sqrt{s_{23}}$. 


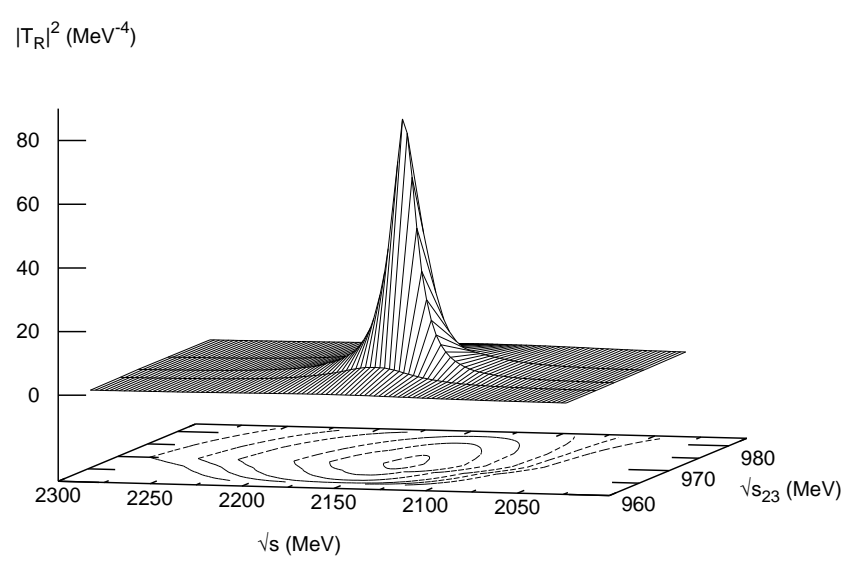

Fig. 8. The $\phi K \bar{K}$ squared amplitude in the isospin 0 configuration.

In this latter work we also evaluated the quantitative effect of cancellation of the off-shell part of the amplitudes with the three body forces. If the later are omitted, keeping the off shell dependence of the amplitudes, the peak still appears but shifted by $40 \mathrm{MeV}$ at lower energies. The cancellation between the off shell terms of the amplitudes and the three body forces has thus a nontrivial consequence if omitted. If we look at it from a different perspective, we can say that should one had used the full off shell extrapolation of the amplitudes one would have to add the effects of the three body force, which in this case would induce a shift of $40 \mathrm{MeV}$ in the mass of the resonance.

To conclude, we are finding a new picture for the low lying $1 / 2^{+}$baryon states with $S=-1$, which largely correspond to bound states or resonances of two mesons and a baryon. In the $S=0$ sector we find a clear signal for the $N^{*}(1710)$, which has a very large branching ratio for decay into $\pi \pi N$ in the PDG, but not for the $N^{*}(1440)$. This negative result for the Roper should rather be interpreted in positive terms as a clear indication that the $\pi \pi N$ that we study is not the most important component of the Roper structure, hinting, together with the study of 37 , at a very complex structure for the $N^{*}(1440)$. In the three meson sector a clear peak could be seen for the three body interacting system $\phi K \bar{K}$, strongly correlated around the $\phi f_{0}(980)$, reflecting the strong coupling of the $f_{0}(980)$ resonance to $K \bar{K}$, the main building block of the $f_{0}(980)$ as a dynamically generated resonance. 


\section{Acknowledgments}

L. S. Geng wishes to acknowledge support from the Ministerio de Educacion in the program of Doctores y Tecnologos extranjeros and D. Strottman in the one of sabbatical. A. Martinez and D. Gamermann from the Ministry of Education and Science and K. Khemchandani from the HADRONTH project for the EU. This work is partly supported by DGICYT Contract No. BFM2003-00856, FPA2007-62777 and the E.U. FLAVIAnet network Contract No. HPRN-CT-2002-00311. This research is part of the EU Integrated Infrastructure Initiative Hadron Physics Project under Contract No. RII3-CT-2004-506078. The work of M. N. was supported by CONACyT México under project 50471-F.

\section{References}

1. J. Gasser and H. Leutwyler, Nucl. Phys. B 250, 465 (1985).

2. U. G. Meissner, Rept. Prog. Phys. 56, 903 (1993) .

3. J. A. Oller and E. Oset, Nucl. Phys. A 620, 438 (1997) [Erratum-ibid. A 652, 407 (1999)] .

4. J. A. Oller and E. Oset, Phys. Rev. D 60 (1999) 074023 arXiv:hep-ph/9809337.

5. J. A. Oller, E. Oset and J. R. Pelaez, Phys. Rev. D 59, 074001 (1999) [Erratum-ibid. D 60, 099906 (1999)] .

6. J. R. Pelaez, Phys. Rev. Lett. 92 (2004) 102001 arXiv:hep-ph/0309292.

7. N. Kaiser, P. B. Siegel and W. Weise, Nucl. Phys. A 594 (1995) 325.

8. E. Oset and A. Ramos, Nucl. Phys. A 635 (1998) 99 .

9. J. A. Oller and U. G. Meissner, Phys. Lett. B 500 (2001) 263.

10. C. Garcia-Recio, M. F. M. Lutz and J. Nieves, Phys. Lett. B 582 (2004) 49.

11. D. Jido, J. A. Oller, E. Oset, A. Ramos and U. G. Meissner, Nucl. Phys. A 725 (2003) 181 .

12. J. A. Oller, Eur. Phys. J. A 28 (2006) 63 arXiv:hep-ph/0603134.

13. E. E. Kolomeitsev and M. F. M. Lutz, Phys. Lett. B 585 (2004) 243.

14. S. Sarkar, E. Oset and M. J. Vicente Vacas, Nucl. Phys. A 750 (2005) 294 [Erratumibid. A $\mathbf{7 8 0}$ (2006) 78] .

15. C. Garcia-Recio, J. Nieves and L. L. Salcedo, Phys. Rev. D 74 (2006) 034025 .

16. M. F. M. Lutz and E. E. Kolomeitsev, Nucl. Phys. A 730 (2004) 392 .

17. L. Roca, E. Oset and J. Singh, Phys. Rev. D 72, 014002 (2005) .

18. E. E. Kolomeitsev and M. F. M. Lutz, Phys. Lett. B 582 (2004) 39 .

19. J. Hofmann and M. F. M. Lutz, Nucl. Phys. A 733 (2004) 142 .

20. F. K. Guo, P. N. Shen, H. C. Chiang and R. G. Ping, Phys. Lett. B 641 (2006) 278 arXiv:hep-ph/0603072.

21. D. Gamermann, E. Oset, D. Strottman and M. J. Vicente Vacas, Phys. Rev. D 76 (2007) 074016 .

22. F. K. M. Guo, P. N. M. Shen and H. C. M. Chiang, Phys. Lett. B 647 (2007) 133 .

23. D. Gamermann and E. Oset, Eur. Phys. J. A 33 (2007) 119.

24. L. S. Geng, E. Oset, L. Roca and J. A. Oller, Phys. Rev. D 75 (2007) 014017.

25. C. Daum et al. [ACCMOR Collaboration], Nucl. Phys. B 187, 1 (1981).

26. J. Hofmann and M. F. M. Lutz, Nucl. Phys. A 763 (2005) 90 .

27. T. Mizutani and A. Ramos, Phys. Rev. C 74 (2006) 065201.

28. H. Walliser, Nucl. Phys. A 548 (1992) 649.

29. K. Abe et al. [Belle Collaboration], arXiv:0708.3812 [hep-ex]. 
30. D. Gamermann and E. Oset, Eur. Phys. J. A, arXiv:0712.1758 [hep-ph].

31. L. Y. Glozman and D. O. Riska, Phys. Rept. 268 (1996) 263 arXiv:hep-ph/9505422.

32. A. Martinez Torres, K.P. Khemchandani and E. Oset, Eur. Phys. J. A35 (2008) 295.

33. A. Martinez Torres, K. P. Khemchandani and E. Oset, Phys. Rev. C 77 (2008) 042203 arXiv:0706.2330 [nucl-th]].

34. T. Inoue et. al., Phys. Rev. C 65. 035204 (2002).

35. W.-M. Yao et al., J. Phys. G 33, 1 (2006).

36. K. P. Khemchandani, A. M. Torres and E. Oset, arXiv:0804.4670 [nucl-th].

37. O. Krehl, C. Hanhart, S. Krewald and J. Speth, Phys. Rev. C 62 (2000) 025207 arXiv:nucl-th/9911080.

38. B. Aubert et al. [BABAR Collaboration], Phys. Rev. D 74 (2006) 091103 arXiv:hep-ex/0610018.

39. B. Aubert et al. [BABAR Collaboration], Phys. Rev. D 76 (2007) 012008.

40. M. Ablikim et al. [BES Collaboration], Phys. Rev. Lett. 100 (2008) 102003.

41. A. Martinez Torres, K. P. Khemchandani, L. S. Geng, M. Napsuciale and E. Oset, arXiv:0801.3635 [nucl-th]. 


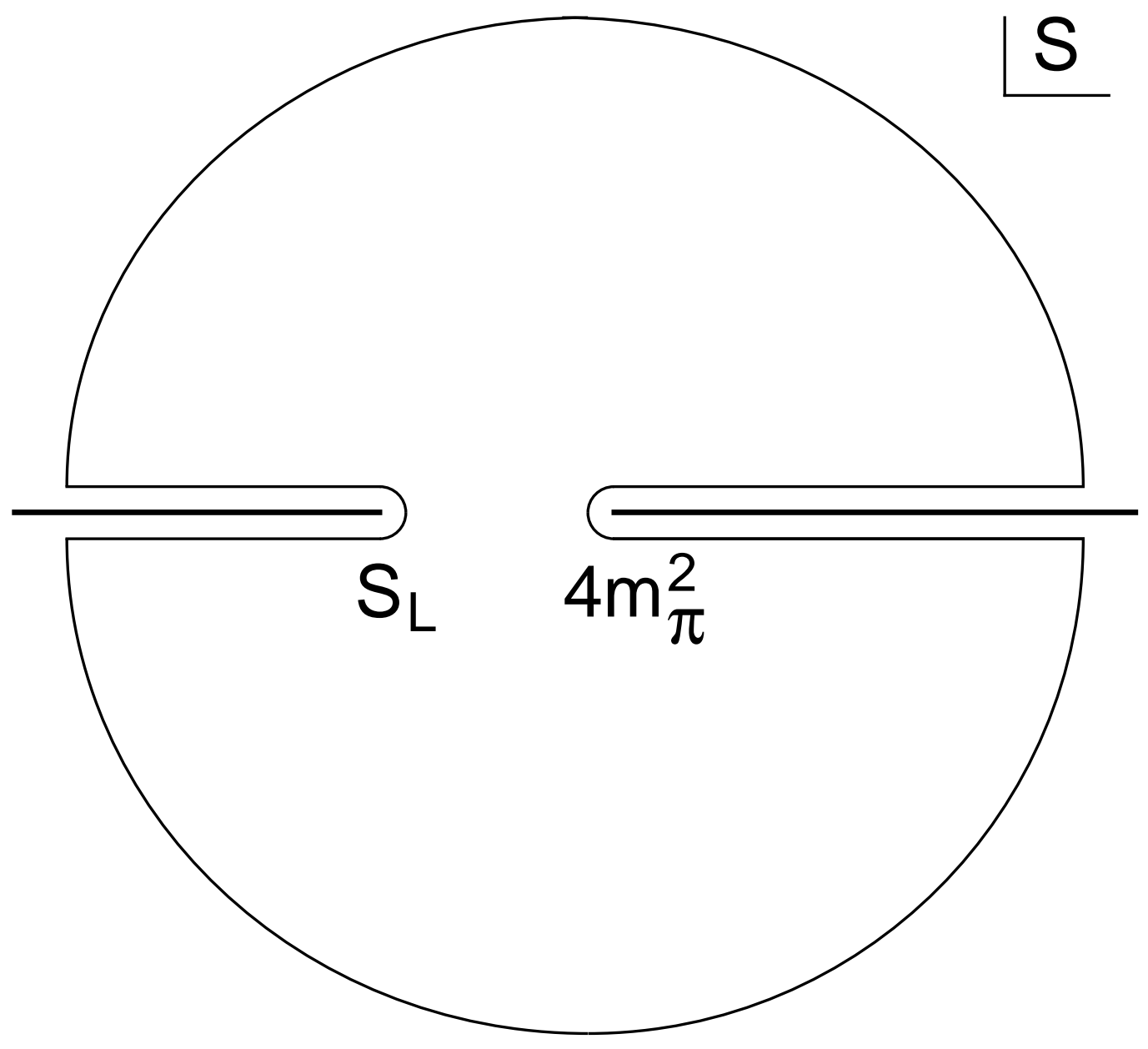




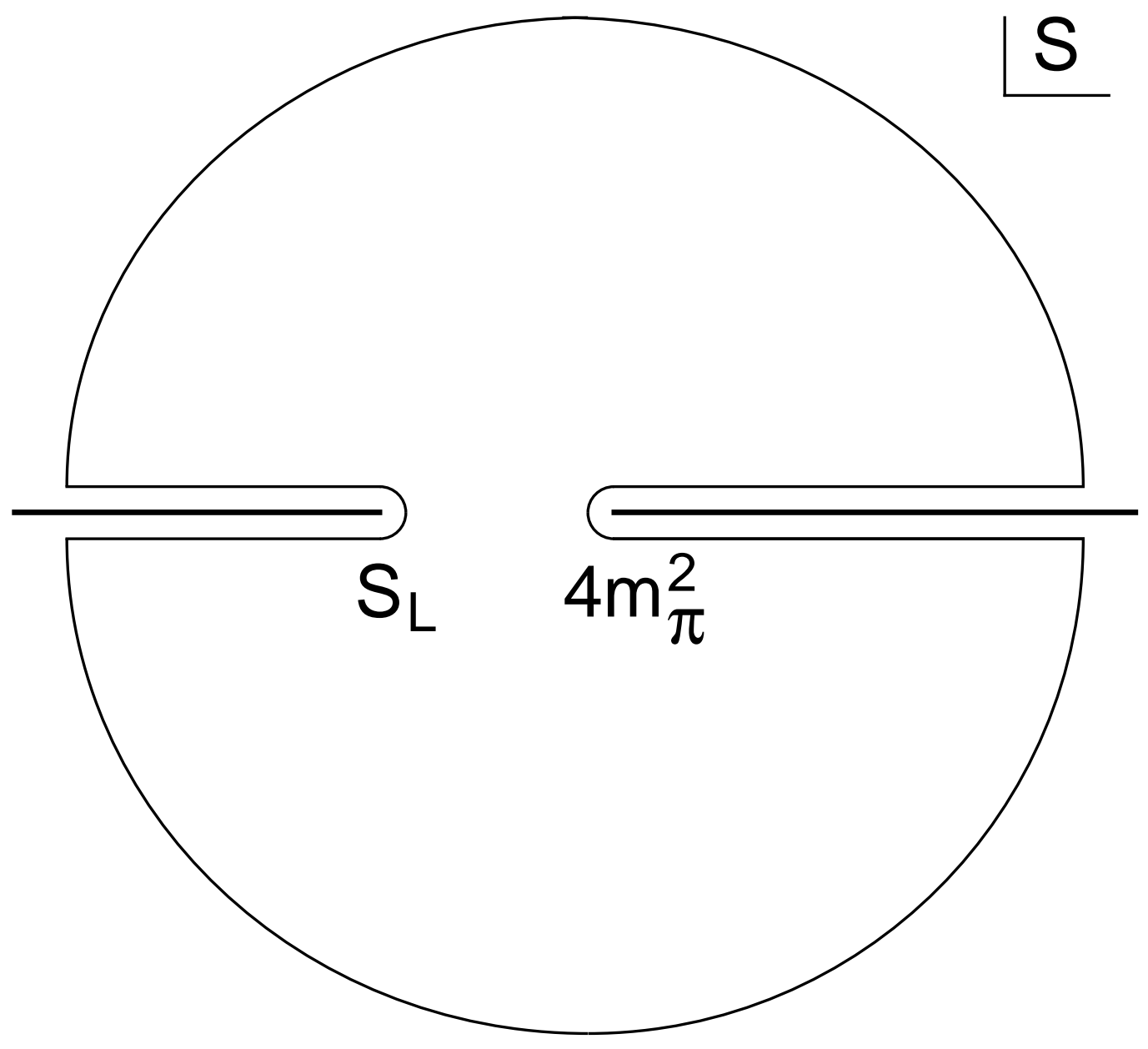

